\title{
Effect of Silane Pressure on Silane-Hydrogen RF Glow Discharges
}

\author{
FRANK J. KAMPAS AND MARK J. KUSHNER, MEMBER, IEEE
}

\begin{abstract}
The intensity and time dependence of optical emission from silane and silane-hydrogen radio-frequency (RF) discharges have been measured as a function of silane pressure $(0.05-1.0$ torr). The rate of emission of $\mathrm{H}^{*}$ and $\mathrm{Si}^{*}$ resulting from dissociative excitation was found to decrease with increasing silane pressure in a manner consistent with a similar decrease in average electron energy. Results from a Monte Carlo plasma simulation code were used to compute the rate of optical emission. Comparison of theory and experiment shows good agreement for emission intensities and confirms for discharges operating at constant pressure and power density a decrease in electron density and average electron energy with increasing silane partial pressure in mixtures of silane and hydrogen. The time-dependent spatially averaged emission intensity of $\mathrm{H}^{*}$ is experimentally nonsymmetric with a shape that is a systematic function of silane partial pressure. This systematic behavior is reproduced by the plasma simulation and is attributed to the change in the dc bias of the powered electrode, which becomes less negative with increasing silane partial pressure.
\end{abstract}

\section{INTRODUCTION}

$\mathrm{T}$ HE DEPOSITION of hydrogenated amorphous silicon $(\mathrm{a}-\mathrm{Si}: \mathrm{H})$ from radio-frequency (RF) glow discharges in silicon containing gases involves a number of deposition parameters including frequency and power of the electrical excitation, deposition system geometry, pressure and flow rate of process gases, and substrate temperature. These factors all influence the flux of molecules, radicals, ions, and photons upon the substrate, which in turn determines the a-Si : $\mathrm{H}$ film properties. The relationship between film properties and deposition parameters can be divided into three interrelated parts: discharge physics, plasma chemistry, and the film growth processes. This paper is concerned with one aspect of the discharge physics, by which we mean the creation, loss, and interactions of free electrons within the discharge. Optical emission spectroscopy was used to characterize the effect of gas pressure on the electron-impact dissociative excitation of $\mathrm{H}^{*}$ and $\mathrm{Si}^{*}$ in discharges in silane and in mixtures of silane and hydrogen. Both time-averaged and time-dependent measurements were made. The systematic behavior of the average electron energy and den-

Manuscript received July 17, 1985; revised October 4, 1985. The work of F. J. Kampas was supported by the Office of Basic Energy Sciences, Division of Materials Science of the U.S. Department of Energy. The work of M. J. Kushner was supported by Spectra Technology, Inc.

F. J. Kampas was with the Metallurgy and Materials Science Division of Brookhaven National Laboratory, Upton, NY 11973. He is now with Chronar, Inc., Princeton, NJ 08540.

M. J. Kushner is with Spectra Technology, Inc. (formerly Mathematical Sciences Northwest, Inc.), Bellevue, WA 98004.

IEEE Log Number 8406832 sity is inferred from the results of these measurements. A Monte Carlo plasma simulation code was used to compute the rate of optical emission from the discharge for similar conditions. The systematic dependence of average electron energy and density was also computed. Comparison of the computed data to the experimental results yielded good agreement and aided in the interpretation of the experimental data.

In Section II, the optical emission processes are discussed and the manner in which we used the optical emission to characterize the plasma is reviewed. The experimental apparatus and procedures are discussed in Section III, and the plasma simulation model is reviewed in Section IV. The experimental and model results are discussed in Section V, and concluding remarks are in Section VI.

\section{Physical Emission Mechanisms}

Electron-impact excitation of molecular hydrogen in the discharges of interest can result in optical emission in one of two ways:

$$
\begin{aligned}
e+\mathrm{H}_{2} & \rightarrow \mathrm{H}_{2}^{*}+e \\
& \rightarrow \mathrm{H}+\mathrm{H}^{*}+e .
\end{aligned}
$$

Optical emission due to molecular hydrogen in a silane or silane-hydrogen discharge results dominantly from excitation of ground-state $\mathrm{H}_{2}$. Dissociative excitation of molecular hydrogen produces emission from a variety of excited states in atomic hydrogen [1]. Similarly, dissociative excitation of silane results in emission from $\mathrm{Si}, \mathrm{SiH}$, and $\mathrm{H}[2]$ :

$$
\begin{gathered}
e+\mathrm{SiH}_{4} \rightarrow e+\mathrm{Si}^{*} \text { or } \mathrm{SiH}^{*} \\
\quad \text { or } \mathrm{H}^{*}+\text { other fragments. }
\end{gathered}
$$

The rate of emission from a particular state $A, I_{A}$, is equal to the time-averaged, or instantaneous, value of $N k_{A} n_{e}$, where $n_{e}$ is the electron density, $k_{A}$ is the electron-impact rate constant, and $N$ is the number density of the parent molecule. Therefore the normalized emissions $I_{\mathrm{Si}} /\left[\mathrm{SiH}_{4}\right]$, $I_{\mathrm{SiH}} /\left[\mathrm{SiH}_{4}\right], I_{\mathrm{H}_{2}} /\left[\mathrm{H}_{2}\right]$ are proportional to their respective products $k_{A} n_{e}$ [3]. By assuming that the deposition rate is proportional to the rate of production of the appropriate free radicals, it can be shown that the deposition rate/ [ $\left.\mathrm{SiH}_{4}\right]$ (normalized deposition rate) is also proportional to a quantity of the form $k_{A} n_{e}$ [3]. The excitation rate constant depends on the electron energy distribution function 
and the energy-dependent electron-impact cross sections. Since the energy dependence of the electron-impact cross sections is known, by taking ratios of the normalized emission intensities to eliminate the electron density, it is possible to determine the effect of deposition parameters on the ratios of the rate constants and therefore determine whether the electron energy distribution is shifting toward higher or lower energy.

Time-resolved optical emission provides information on the mechanisms by which energy is transferred from the $\mathrm{RF}$ electric field to the electrons in the discharge. The fact that the optical emission from silane RF discharges is time and spatially dependent was first shown by de Rosny $e t$ al. [4]. By spatially and temporally resolving the optical emission, they showed that for their reactor geometry, emission results from an excitation wave which propagates from the electrodes.

\section{Experimental Apparatus and Procedures}

Silane-hydrogen mixtures at 1.0-torr pressure were studied while operating at constant RF power of $30 \mathrm{~W}$ $\left(0.017 \mathrm{~W} \cdot \mathrm{cm}^{-3}\right)$. The RF frequency was $13.56 \mathrm{MHz}$. The applied RF voltage was varied to maintain the constant power condition (RF amplitude 65-95 V). The dc bias of the powered electrode was simultaneously measured. The capacitively coupled RF discharge, having electrodes $25 \mathrm{~cm}$ in diameter separated by $3.5 \mathrm{~cm}$, is described in detail elsewhere [5]. Pure silane discharges were studied in a smaller system with $10-\mathrm{cm}$ electrodes separated by $3.0 \mathrm{~cm}$. Both systems were asymetric in that the grounded electrode was connected to the metal vacuum chamber, thereby increasing its effective area.

Light emitted from the discharges was dispersed by a Spex model 1400 0.75-m monochrometer and detected with a thermoelectrically cooled Hamamatsu R955P photomultiplier tube (PMT). Emission intensities were obtained with a photon counting system consisting of a Photochemical Research Associates model 1763 preamplifier and 1762 amplifier/discriminator, and a Canberra model 2071 counter/timer.

Time-resolved emission spectra from the silane-hydrogen mixtures were obtained using a Canberra model 2044 time-to-amplitude converter (TAC). The discriminated PMT output from the amplifier/discriminator was connected to the start-pulse input of the TAC. The RF voltage applied to the deposition system was attenuated and processed by a second amplifier/discriminator to generate the stop pulse for the TAC. The output of the TAC was analyzed by a Nuclear Data model 62 multichannel analyzer (MCA). A similar system, consisting of an Ortec Adcam multichannel buffer interfaced to an Apple IIe computer and an Ortec model $467 \mathrm{TAC}$, was used for the study of the pure silane discharges.

\section{Plasma Simulation Model}

A plasma simulation model was used to aid in the interpretation of the experimental data. A Monte Carlo particle simulation technique is used to compute the time- and spatially dependent electron distribution function between parallel plates of a capacitively coupled RF discharge. While doing so, the time- and spatially dependent rates of electron collision excitation of the gas are also obtained. The model is described in detail elsewhere [6]-[8] and therefore only the features particular to this application will be discussed.

In applying the plasma simulation to this study, mixtures of $\mathrm{H}_{2}$ and $\mathrm{SiH}_{4}$ were modeled. Electron collisions cross sections for $\mathrm{H}_{2}$ were obtained from [1], [9], and [10], and cross sections for $\mathrm{SiH}_{4}$ were obtained from [11][13]. The dissociative excitation of $\mathrm{H}_{2}$, yielding atomic emission from $\mathrm{H}$ at $656 \mathrm{~nm},\left(\mathrm{H}_{\alpha}\right)$ was collated from [1] and [14], while that for dissociative excitation of $\mathrm{SiH}_{4}$ was taken from [2]. Inputs to the plasma simulation are the gas mixture, pressure, RF frequency, amplitude of the $\mathrm{RF}$ voltage, and the value of the dc bias on the powered electrode.

The quantity of interest for comparison of results from the plasma simulation to experiment is the excitation parameter $k_{A} n_{e}$. The electron-impact rate constant for process $A, k_{A}$, is obtained directly from the simulation by time and spatially averaging the computed electron distribution function and convolving it with the product of $v_{e} \sigma\left(v_{e}\right)$, where $v_{e}$ is the electron velocity and $\sigma\left(v_{e}\right)$ is the electron-impact excitation cross section. Since the plasma simulation does not at present directly compute an electron density, this value is obtained indirectly in the following fashion. From the computed electron distribution function, one calculates the electron-impact rate constants for all elastic and inelastic collisions. The total rate of energy loss of the average electron $P_{e}$ is obtained by summing the product of the inelastic rate coefficients, collision partner number density, and the energy loss per collision $\Delta \epsilon$ and adding the elastic energy loss rate

$$
P_{e}=\sum N_{i} k_{i} \Delta \epsilon_{i}+\sum K_{j}^{E}
$$

The rate of energy loss resulting from elastic collision $j$, $K_{j}^{E}$, is given by

$$
K_{j}^{E}=\int_{0}^{\infty}\left[\frac{2 N \epsilon m_{e}}{M}\right] \sigma(\epsilon)\left[\frac{2 \epsilon}{m_{e}}\right]^{1 / 2} f(\epsilon) \epsilon^{1 / 2} d \epsilon
$$

where $\epsilon$ is the electron energy, $f(\epsilon)$ is the electron distribution function, $N$ is the density of the heavy particle collision partner, and $m_{e}$ and $M$ are the masses of the electron and heavy particle, respectively. Given the total RF power deposited in the plasma measured experimentally for the same conditions, $P_{R F}$, and the volume of the plasma $V$, the electron density is obtained from $n_{e}=P_{R F} /\left(P_{e} V\right)$.

\section{Results And Discussion}

Normalized emission intensities for $\mathrm{Si}(288 \mathrm{~nm}), \mathrm{H}_{2}$ (continuum emission near $288 \mathrm{~nm})$, and $\mathrm{H}^{*}\left(\mathrm{H}_{\alpha^{\prime}} 656 \mathrm{~nm}\right)$, and the normalized deposition rate are shown in Fig. 1 for a $30-\mathrm{W}\left(0.017 \mathrm{~W} \cdot \mathrm{cm}^{-3}\right)$ silane-hydrogen discharge as a function of silane fraction. The total pressure was maintained at 1 torr, and the total flow rate was held constant 


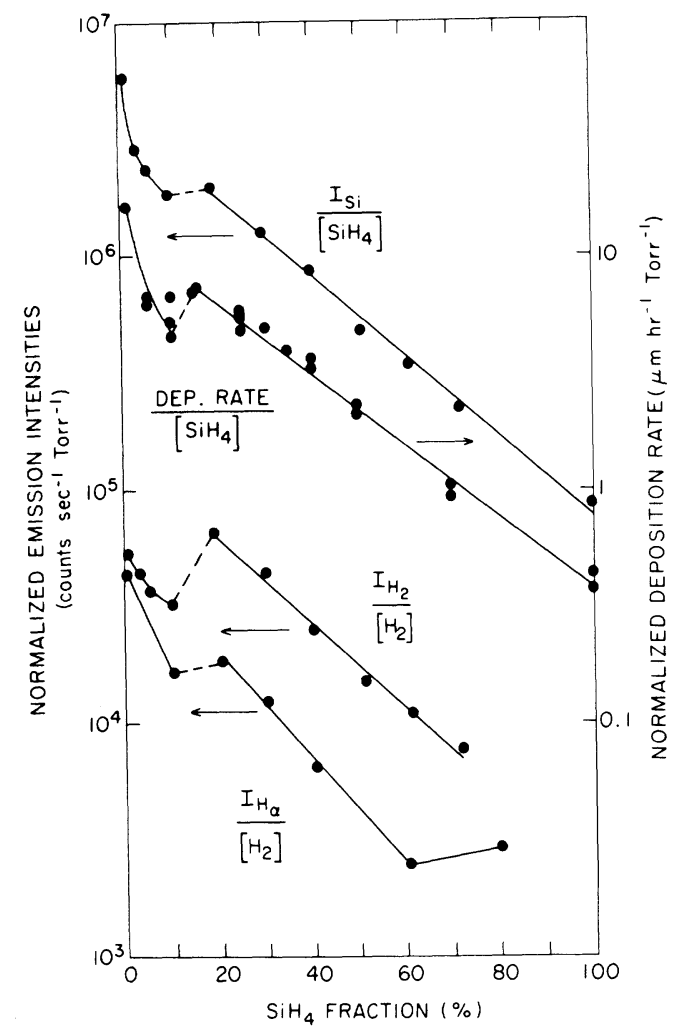

Fig. 1. Normalized emission intensities and normalized deposition rates for a 1.0-torr mixture of $\mathrm{SiH}_{4}$ and $\mathrm{H}_{2}$. The discharge power was held constant $\left(0.017 \mathrm{~W} \cdot \mathrm{cm}^{-3}\right)$ by varying the applied $\mathrm{RF}$ voltage. The more rapid decrease in $I_{\mathrm{H}}$ as compared to $I_{\mathrm{Si}}$ with increasing $\mathrm{SiH}_{4}$ fraction implies a decrease in average electron energy.

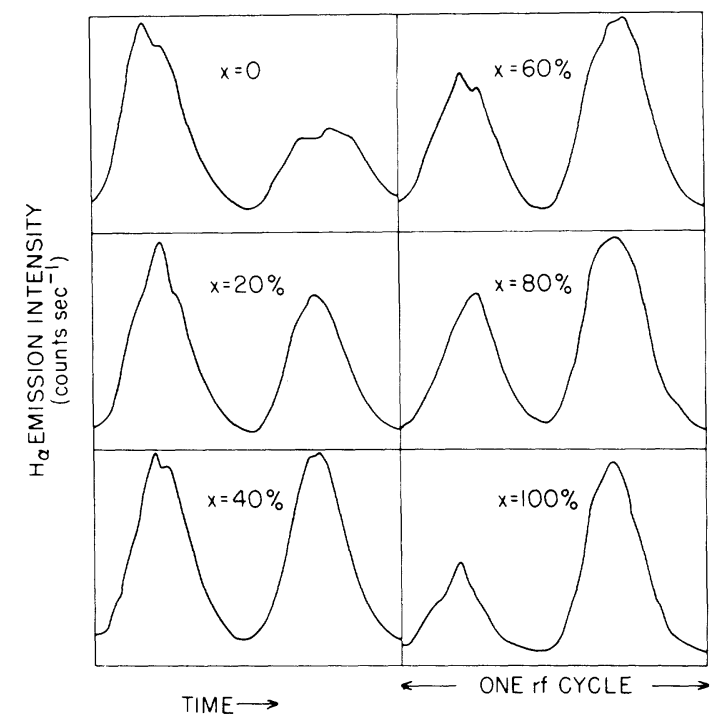

Fig. 2. $\mathrm{H}_{x x}$ emission intensity as a function of time and silane mole fraction ( $x$ ) for discharges in mixtures of $\mathrm{SiH}_{4}$ and $\mathrm{H}_{2}$. The RF frequency is 13.56 $\mathrm{MHz}$ and the conditions are identical to those for Fig. 1. The change in the relative amplitude of the two peaks in emission can be correlated to a change in the electrode having the largest sheath potential.

at $100 \mathrm{sccm}$. Time-resolved atomic $\mathrm{H}^{*}$ optical emission is shown in Fig. 2, also as a function of silane fraction. The normalized emission intensities and deposition rates decrease exponentially with increasing silane fraction, indicating that the products $k_{A} n_{e}$ are also decreasing.

In mixtures of silane and hydrogen, $\mathrm{H}^{*}$ emission at 656 $\mathrm{nm}$ can be obtained by dissociative excitation of both $\mathrm{H}_{2}$

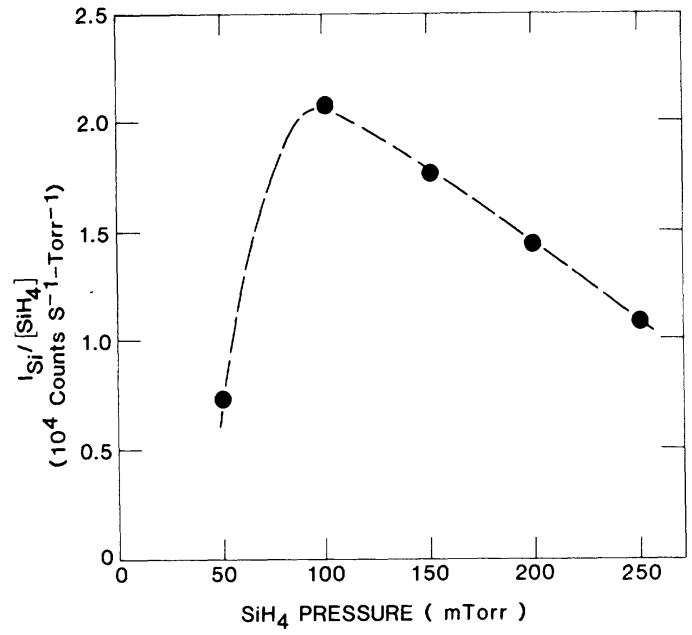

Fig. 3. Normalized Si emission as a function of pressure for RF discharges in silane. The applied RF voltage was held constant (amplitude $100 \mathrm{~V}$ ).

and $\mathrm{SiH}_{4}$. The cross section for this process for both molecules has a threshold of approximately $16.1 \mathrm{eV}$. For purposes of obtaining the normalized emission rate, though, we have assumed that the majority of $\mathrm{H}^{*}$ emission results from dissociative excitation of $\mathrm{H}_{2}$ and normalized $\mathrm{H}^{*}$ as $I_{\mathrm{H}} /\left[\mathrm{H}_{2}\right]$. In doing so, the normalized intensity for $\mathrm{H}^{*}$ is artificially large at small fractions of $\mathrm{H}_{2}$ because some of the observed $\mathrm{H}^{*}$ emission results from dissociative excitation of $\mathrm{SiH}_{4}$. In spite of this normalization, $I_{\mathrm{H}} /\left[\mathrm{H}_{2}\right]$ decreases most rapidly with increasing silane pressure. When comparing this value to the normalized Si emission, whose threshold for excitation by electron impact is $9.5 \mathrm{eV}$, and the normalized $\mathrm{H}_{2}$ emission, whose threshold for excitation by electron impact is $11.9 \mathrm{eV}$, we can conclude that the electron distribution shifts toward lower energies with increasing silane fraction [3].

The time-resolved $\mathrm{H}^{*}$ emission shows two peaks whose relative amplitudes reversed with increasing silane fraction. This reversal experimentally correlates with the self$\mathrm{dc}$ bias, which was negative for low silane fraction $(-40$ $\mathrm{V}$ for 1-percent $\mathrm{SiH}_{4}$ ), but increased and reversed sign as the silane fraction increased ( $+5 \mathrm{~V}$ for 100-percent $\mathrm{SiH}_{4}$ ). The increase in dc bias is consistent with there being a corresponding increase in the density of negative ions as the fraction of silane increases [15].

Normalized Si emission and time-resolved Si emission are shown for a pure silane discharge as a function of pressure in Figs. 3 and 4, respectively. For these measurements, the applied RF voltage was held constant (amplitude $100 \mathrm{~V}$ ). The normalized Si emission decreases with increasing silane pressure and the time-resolved emission has two peaks. Qualitatively, the results are similar to the silane-hydrogen mixture, except for the low silane pressure, 0.05 torr. At that pressure, only one peak appeared in the time-resolved emission, and the emission intensity was low as a result. The relative amplitude of the first peak of the time-resolved emission increases compared to the second with increasing silane pressure. Again, this change in relative amplitude of the emission peaks can be correlated with an increase in the dc bias of 


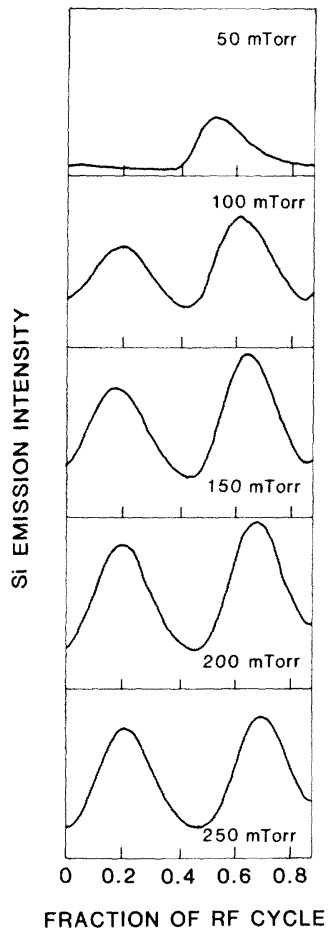

Fig. 4. Time-resolved Si emission for the conditions of Fig. 3. The time axis corresponds to 0.86 of the RF cycle $(13.56 \mathrm{MHz})$.

the powered electrode with increasing silane pressure $(-16$ to $-4 \mathrm{~V})$.

Results from the plasma simulation for electron density and the rate of $\mathrm{H}^{*}$ emission for constant power RF discharges in 1.0-torr silane-hydrogen mixtures appear in Fig. 5(a). The experimentally measured values of applied RF voltage, RF power, and dc bias were used as input parameters for the simulations. The computed rate of $\mathrm{H}^{*}$ emission is the product

$$
I_{\mathrm{H}}=n_{e}\left[k_{\mathrm{H}_{2}} f_{\mathrm{H}_{2}}+k_{\mathrm{SiH}_{4}} f_{\mathrm{SiH}_{4}}\right]
$$

where $k_{A}$ is the electron-impact dissociative excitation rate constant for molecule $A$ and $f_{A}$ is the mole fraction of that molecule. The electron density, a volume-averaged value, decreases from $6.4 \times 10^{9} \mathrm{~cm}^{-3}$ to $3.6 \times 10^{9} \mathrm{~cm}^{-3}$ as the partial pressure of silane increases from 0 to 1.0 [16]. ${ }^{1}$ The computed rate of $\mathrm{H}^{*}$ emission also decreases with increasing fractions of $\mathrm{SiH}_{4}$, though to a much more severe degree. For comparison with the experiment, we renormalized the computed rate of emission as $I_{\mathrm{H}}^{\prime}=I_{\mathrm{H}} / f_{\mathrm{H}_{2}}$. The rate of emission is plotted in Fig. 5(b). The corresponding experimental data are also plotted in Fig. 5(b) as the open triangles. The agreement is quite good. The upturn in the experimental value for the rate of $\mathrm{H}^{*}$ emission is not a result of an increase in the actual rate but rather a result of the normalization by $\left[\mathrm{H}_{2}\right]$.

${ }^{1}$ Measurements of the electron density of $\mathrm{dc}$ and pulsed hollow cathode discharges sustained in $\mathrm{He} / \mathrm{SiH}_{4}$ mixtures have recently been made using microwave interferometry. The electron density was observed to decrease $\left(12 \rightarrow 2 \times 10^{9} \mathrm{~cm}^{-3}\right)$ with increasing $\mathrm{SiH}_{4}$ mole fraction $(0.01<M<$ $0.04)$. The electron density obtained here by energy balance in $\mathrm{H}_{2} / \mathrm{SiH}_{4}$ mixtures, $6-4 \times 10^{9} \mathrm{~cm}^{-3}\left(P=17 \mathrm{~mW} \cdot \mathrm{cm}^{-3}\right)$, compares favorably with those experimentally measured for similar $\mathrm{RF}$ power deposition $(\mathrm{P} \approx 15$ $\mathrm{mW} \cdot \mathrm{cm}^{-3}$ ) in $\mathrm{He} / \mathrm{SiH}_{4}$ mixtures.

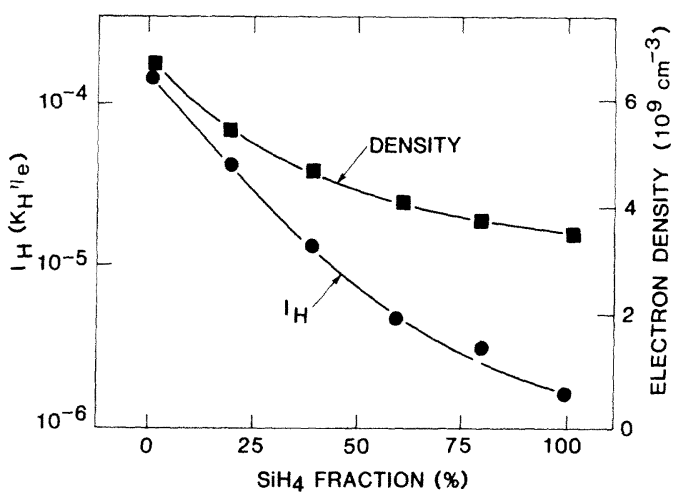

(a)

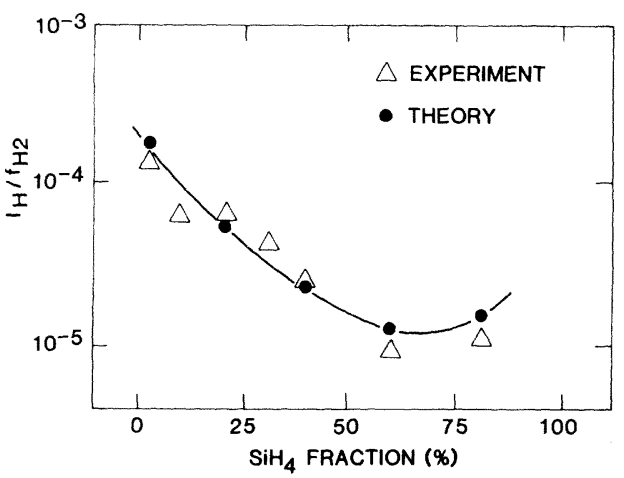

(b)

Fig. 5. Results from the plasma simulation for normalized $\mathrm{H}_{\alpha}$ emission intensity and electron density for mixtures of $\mathrm{SiH}_{4}$ and $\mathrm{H}_{2}$. The conditions are the same as for Fig. 1. The normalized emission intensity in (a) is the product of total excitation rate constant times electron density. The emission intensity in (b) has the same normalization as the experimental data, also shown as the open triangles.

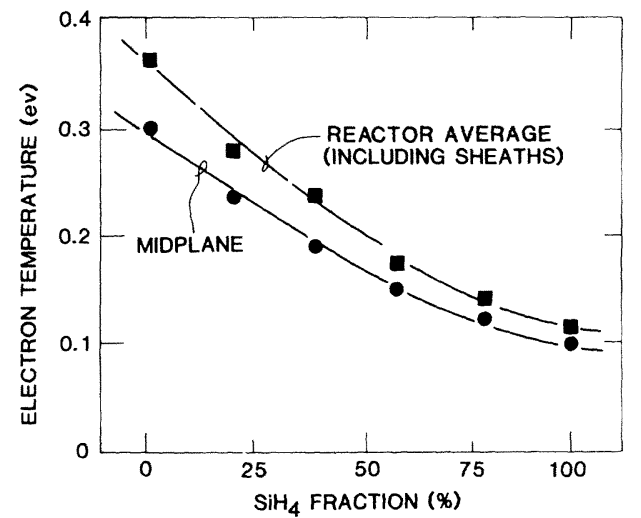

Fig. 6. Results from the plasma simulation for electron temperature ( $T_{c}=$ $2 \epsilon / 3 k, \epsilon=$ average electron energy) for the conditions of Fig. 1. The volume-averaged electron temperature and the electron temperature and the midplane of the reactor are shown. The difference in temperature is due to the contribution of energetic electrons near the sheath boundary.

Recall in the discussion of the experimental results that the rate of $\mathrm{H}^{*}$ emission $k_{\mathrm{H}} n_{e}$ decreased more rapidly than the rate of $\mathrm{Si}^{*}$ emission $k_{\mathrm{Si}} n_{e}$ as the fraction of $\mathrm{SiH}_{4}$ increased. Due to the lower energy threshold for $\mathrm{Si}^{*}$ emission, one concludes that the average electron energy also decreases with increasing $\mathrm{SiH}_{4}$ fraction. This conclusion is confirmed with results from the plasma simulation, plotted in Fig. 6, where the average electron temperature is plotted as a function of the silane fraction. The average electron temperature decreases with increasing $\mathrm{SiH}_{4}$ frac- 


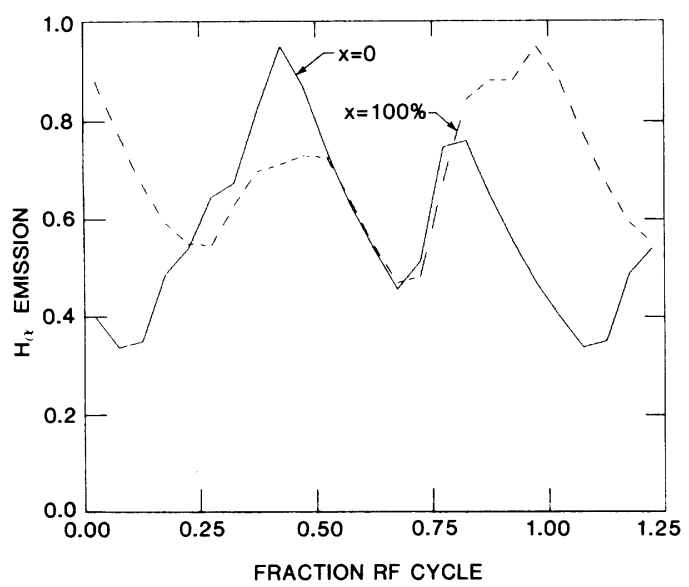

Fig. 7. Time-resolved $\mathrm{H}_{\alpha}$ emission as computed with the plasma simulation for the conditions of Fig. 2. Results for silane mole fractions of $x$ $=0$ and $x=100$ percent are plotted. The reversal in the phase of the maximum emission observed experimentally is reproduced, confirming the correlation of the phase of the maximum emission with the electrode having the maximum sheath potential.

tion $\left(T_{e}=2 \epsilon / 3 k, \epsilon=\right.$ average electron energy). Two values of the average electron temperature are plotted: average temperature for the spatial location at the midplane between the electrodes, and electron temperature averaged over the entire volume of the discharge. The midplane value is lower than the reactor averaged value; the discrepancy is due to the contribution to the average electron energy of high-energy electrons resulting from secondary electron emission from the electrodes and from the acceleration of low-energy electrons by the oscillating sheaths [6]-[8]. Due to the high pressure of the discharges in these examples (1.0 torr), the range of high-energy electrons accelerated by processes associated with the sheaths is only a few millimeters. The high-energy component of the electron distribution function is constrained to a region close to the electrodes, thereby accounting for the difference in total average electron energy and that at the center of the discharge. The high-energy contribution to the average electron energy decreases from 17 to 11 percent with increasing $\mathrm{SiH}_{4}$ fraction.

Simulated time-dependent spatially averaged rates of emission of $\mathrm{H}^{*}$ are plotted in Fig. 7. The conditions are similar to those for the experimental results for time-dependent emission plotted in Fig. 2. The simulated rates of emission are obtained from the computed time-dependent excitation rates by integrating over the lifetime of previously excited atoms

$$
I_{\mathrm{H}}(t) \propto \int_{-\infty}^{t} k_{\mathrm{H}}\left(t^{\prime}\right) e^{-\left(t-t^{\prime}\right) / \tau_{r}} d t^{\prime}
$$

where $\tau_{r}$ is the radiative lifetime of the excited state. The two peaks in amplitude, as observed experimentally, are qualitatively reproduced as is the time reversal in their appearance with increasing silane mole fraction. The time reversal results from the change in the dc bias on the powered electrode-negative for small silane mole fraction and positive for large silane mole fraction. Due to the relatively large threshold energy for dissociative excitation and the low plasma temperature, the rate of emission is sensitive to the high-energy nonthermal tail of the electron distribution function. High-energy electrons result from secondary electron emission at the electrodes and from electrons in the plasma near the electrodes being accelerated by the oscillating sheath potential. The change in the dc bias from negative to positive changes the location, and hence relative phase, of the maximum sheath potential from the powered electrode to the grounded electrode. The result is that the phase in the modulation of the high-energy tail of the electron distribution function, and hence rate of excitation of $\mathrm{H}^{*}$, also changes in phase.

\section{Concluding Remarks}

Emission from $\mathrm{H}^{*}$ and $\mathrm{Si}^{*}$ in low-pressure $\mathrm{RF}$ glow discharges has been measured as a function of silane pressure. For discharges in mixtures of $\mathrm{SiH}_{4}$ and $\mathrm{H}_{2}$ where the pressure and RF power are kept constant (1.0 torr, 0.017 $\mathrm{W} \cdot \mathrm{cm}^{-3}$ ), the normalized rate of emission indicates that the average electron temperature decreases with increasing $\mathrm{SiH}_{4}$ mole fraction. The time dependence of the emission has two peaks, one with higher amplitude than the first. The relative phase of the peak with the higher amplitude can be directly correlated to the electrode having the largest sheath potential, as inferred from the measured change in the dc bias of the powered electrode. Results from a plasma simulation code agree well with the experimental measurements and confirm these conclusions. The simulated emission rates were computed based on the input gas concentration to the discharges, thereby ignoring dissociation. It is well known, though, that highly powered $\mathrm{RF}$ discharges in $\mathrm{SiH}_{4}$ undergo significant dissociation [17]. The good agreement obtained between experiment and theory, where the consequences of dissociation are ignored, implies that for the pressures, flow rates, and power deposition of our experiment, significant dissociation of $\mathrm{SiH}_{4}$ does not occur.

\section{REFERENCES}

[1] D. A. Vroom and F. J. De Heer, "Production of excited atoms by impact of fast electrons on molecular hydrogen and deuterium," $J$. Chem. Phys., vol. 50, p. 580, 1969.

[2] J. Perrin and J. P. M. Schmitt, "Emission cross sections from fragments produced by electron impact on silane," J. Chem. Phys., vol. 67 , pp. $167-176,1982$.

[3] F. J. Kampas, "An optical emission study of the glow-discharge deposition of hydrogenated amorphous silicon from argon-silane mixtures," J. Appl. Phys., vol. 54, p. 2276, 1983.

[4] G. de Rosny, E. R. Mosburg, Jr., J. R. Abelson, G. Devaud, and R. C. Kerns, "Evidence for a time dependent excitation process in silane radio frequency glow discharges," J. Appl. Phys., vol. 54, p. 2272, 1983.

[5] P. E. Vanier, F. J. Kampas, R. R. Corderman, and G. Rajeswaran, "A study of hydrogenated amorphous silicon deposited by RF glow discharge in silane-hydrogen mixtures,"' J. Appl. Phys., vol. 56, p. $1812,1984$.

[6] M. J. Kushner, "Monte-Carlo simulation of electron properties in RF parallel plate capacitively coupled discharges,"' J. Appl. Phys., vol. 54, pp. 4958-4965, 1983.

[7] M. J. Kushner, H. Anderson, and P. J. Hargis, Jr., "Simulation of time and spatially dependent excitation processes in RF discharges 
for plasma processing," in Plasma Synthesis and Etching of Electronic Materials, vol. 38, Mater. Res. Soc. Symp. Proc. Pittsburgh, PA: Mater. Res. Soc., 1985, pp. 201-214.

[8] M. J. Kushner, "Mechanisms for power deposition in $\mathrm{Ar} / \mathrm{SiH}_{4}$ capacitively coupled RF discharges,', IEEE Trans. Plasma Sci., vol. PS-14, no. 2, pp. 188-196, Apr. 1986.

[9] M. Hayashi, "Monte Carlo simulation of electron avalanche in hydrogen," J. De Physique (Coll. C7, Suppl. 7), vol. 40, pp. C7/45$46,1979$.

[10] S. J. B. Corrigan, "Dissociation of molecular hydrogen by electron impact," J. Chem. Phys., vol. 43, pp. 4381-4386, 1965.

[11] H. Catham, D. Hils, R. Robertson, and A. Gallagher, "Total and partial electron collision ionization cross sections for $\mathrm{CH}_{4}, \mathrm{C}_{2} \mathrm{H}_{6}$, $\mathrm{SiH}_{4}$, and $\mathrm{Si}_{2} \mathrm{H}_{6}$, , J. Chem. Phys., vol. 81, p. $1770,1984$.

[12] J. Perrin, J. P. M. Schmitt, G. De Rosny, B. Drevillon, J. Huc, and A. Lloret, "Dissociation cross sections for silane and disilane by electron impact,"'J. Chem. Phys., vol. 73, pp. 383-394, 1982.

[13] A. Garscadden, G. L. Duke, and W. F. Bailey, "Electron kinetics of silane discharges,"' Appl. Phys. Lett., vol. 43, p. 1012, 1983.

[14] L. D. Weaver and R. H. Hughes, "Production of $n=3$ and $n=4$ states of atomic hydrogen by electron impact on $\mathrm{H}_{2}$, 'J. Chem. Phys., vol. 52, p. 2299, 1970.

[15] C. A. DeJoseph, Jr., P. D. Haaland, and A. Garscadden, "Basic processes in silane discharges,' in Proc. 1985 IEEE Int. Conf. Plasma Sci. New York: IEEE Press, 1985, p. 13.

[16] C. B. Fleddermann, J. H. Beberman, and J. T. Verdeyen, "Measurement of the electron density and attachment rate coefficient in silane/helium discharges," J. Appl. Phys., vol. 58, pp. 1344-1348, 1985.

[17] C. A. DeJoseph, Jr., and D. R. Pond, "Dissociation rates of silane in an RF discharge by tunable diode laser absorption," presented at the 37th Annu. Gaseous Electron. Conf., 1984, Paper HA-5. 